\title{
Evaluation of risk of malignancy index as a diagnostic tool in cases with adnexal mass
}

\author{
Nidhi Kumari, Vineeta Gupta*, Rashmi Kumari, Amrita Makhija
}

Department of Obstetrics and Gynaecology, Shri Guru Ram Rai Institute of medical and health sciences, Dehradun, India

Received: 22 March 2016

Revised: 29 March 2016

Accepted: 23 April 2016

\author{
*Correspondence: \\ Dr. Vineeta Gupta, \\ E-mail: vineetahims@yahoo.co.in
}

Copyright: (c) the author(s), publisher and licensee Medip Academy. This is an open-access article distributed under the terms of the Creative Commons Attribution Non-Commercial License, which permits unrestricted non-commercial use, distribution, and reproduction in any medium, provided the original work is properly cited.

\begin{abstract}
Background: Ovarian tumour usually presents as adnexal mass but often it is difficult to differentiate between benign and malignant tumour. Several diagnostic modalities such as sonography and tumours markers have been evaluated in the past, but none have been established as an ultimate diagnostic tool individually. The development of a mathematical formula using a logistic model, incorporating menopausal status, the serum level of a glycoprotein called CA-125 and USG score has been described in the form of different malignancy indices. The purpose of this study was to evaluate the various risks of malignancy indices (RMI 1, 2, 3, and 4) in the pre-operative evaluation of adnexal masses especially to differentiate between benign and malignant masses. Another objective of the present study was to compare the four RMI with each other in terms of various statistical parameters like specificity and sensitivity.

Methods: Women with adnexal masses who underwent surgical treatment were included in this study as histopathological examination was taken as gold standard to calculate the accuracy of RMI. The sensitivity, specificity and positive predictive value and negative predictive value of all the four RMI were calculated and data analyzed.

Results: A total of 65 patients were included in the study. RMI 1,2,3,4 was calculated according to their formula. Sensitivity of RMI- 1, 2, 3 and 4 was calculated to be $63.63 \%, 77.27 \%, 63.63 \%$ and $77.27 \%$ respectively. Specificity of RMI- 1, 2, 3 and 4 was calculated to be $69.04 \%, 64.28 \%, 64.28 \%$ and $62.79 \%$ respectively.

Conclusions: Risk of malignancy index is a good diagnostic tool to differentiate between benign and malignant pelvic masses. RMI- 2 and RMI-4 had maximum sensitivity while RMI-1 had maximum specificity. Overall RMI-2 appears to be the most accurate of all the four RMI.
\end{abstract}

Keywords: Risk malignancy index, Adnexal mass, Ovarian tumour

\section{INTRODUCTION}

Ovarian cancer remains the third most frequent gynaecological neoplasm and is a cause of morbidity and mortality worldwide. Recently the age standardized incidence rate for ovarian cancer in India were found to be from 0.9 to 8.4 per 100000 person years among various registries. ${ }^{1}$
Majority of the cases of ovarian cancer are detected at advanced stages. Ovarian tumour usually presents as adnexal mass but often it is difficult to differentiate between benign and malignant tumour. Several diagnostic modalities such as sonography and tumours markers have been evaluated in the past, but none have been established as an ultimate diagnostic tool individually. 
The development of a mathematical formula using a logistic model, incorporating menopausal status, the serum level of a glycoprotein called CA-125 and USG score has been described in the form of different malignancy indices.

These indices were calculated using simplified regression equation obtained from the product of the USG finding score, the menopausal status score and absolute value of CA-125 serum level.

Jacob et al developed a RMI and it is termed RMI- $1{ }^{2}$ Tingulstad et al developed a RMI in 1996 known as RMI2 and they modified it to form the RMI-3. ${ }^{3}$ Yamamoto et al who created their own model of malignancy risk index, added the parameter of tumour size to the RMI and have termed it RMI-4. ${ }^{4}$

The difference between the three indices lies in the different scoring of USG finding and menopausal status.

Cut off value of 200 for RMI-1, 2 and 3 and 450 for RMI-4 showed the best discrimination between benign and malignant pelvic mass with high level of sensitivity and specificity. Main advantage of all the four RMI is that they are simple scoring systems that can be applied directly into clinical practice without the introduction of expensive or complicated methods.

The purpose of this study was to evaluate the various risks of malignancy indices (RMI 1, 2, 3, 4) in the preoperative evaluation of adnexal masses especially to differentiate between benign and malignant masses. Another objective of the present study was to compare the four RMI with each other in terms of various statistical parameters like specificity and sensitivity.

\section{METHODS}

The present prospective study was carried out in the Department of Obstetrics and Gynaecology at SGRR Institute of medical and health sciences, Dehradun after approval and clearance from research and ethics committee of the Institute. Only women with adnexal masses who underwent surgical treatment were included in this study as histopathological examination was taken as gold standard to calculate the accuracy of RMI. An informed consent was obtained from all the patients. After thorough history and clinical examination, patients were subjected to various routine and special investigations.

Serum CA-125 samples were assayed by radio immunoassay. Abnormal CA-125 level is defined as serum levels $>35 \mathrm{U} / \mathrm{ml}$. Transabdominal scans were done using a $3.5 \mathrm{MHz}$ and transvaginal scan done with $7.5 \mathrm{MHz}$ transducer. The lesions were evaluated according to shape, size, and multiplicity, thickness of wall \& septa and ascites. Scoring system based on sonographic findings is shown in Table 1.
Morphological evaluation was performed using the inner wall structure, wall thickness, presence of septa and their thickness and echogenecity.

Menopausal status was noted. Menopausal status was defined as one or more years of amenorrhea or women who had undergone hysterectomy. All other women were considered premenopausal. Risk of malignancy index was calculated according to USG findings, absolute values of CA - 125 serum levels and menopausal status.

Based on the data obtained RMI-1, RM1-2, RMI -3 and RMI-4 were calculated as described below for all patients:

1. $\mathrm{RMI}-1=\mathrm{U}$ X M X CA - 125 value as total USG score of 0 made $U=0$. Score of 1 made $U=1$ and a score of $\geq 2$ made $U=3$. Premenopausal status made $M=1$ and post-menopausal status $M=3$. The serum level of CA-125 was applied directly to the calculation.

2. $\mathrm{RMI}-2=\mathrm{U} X \mathrm{X} X \mathrm{CA}-125$ when a total USG score of 0 or 1 made $U=1$ and score $\geq 2$ made $U=4$. Premenopausal status $\mathrm{M}-1$ and post-menopausal status $\mathrm{M}=4$. The serum level of CA-125 was applied directly to the calculation.

3. RMI-3 = U X M X CA 125 when total USG score of 0 or 1 made $U=1$ and a score of $\geq 2$ made $U=3$. Premenopausal status made $\mathrm{M}=1$ and postmenopausal status $\mathrm{M}=3$. The serum level of $\mathrm{CA}$ 125 was applied directly to the calculation.

4. $\mathrm{RMI}-4=\mathrm{U} X \mathrm{X} X$ Size $(\mathrm{cm}) \mathrm{X} \mathrm{CA}-125$. When total USG score 0 or 1 made $U=1$ and score of $\geq 2$ made $U=4$. Premenopausal status made $M=1$ and postmenopausal $=4$. Tumour size (single greatest diameter) of $\leq 7 \mathrm{~cm}$ made $S=1$ and $\geq 7 \mathrm{~cm}$ made $S=2$. The serum level of CA-125 was applied directly to the calculation.

All the patients either underwent laparotomy or laparoscopy. The mass excised was sent for histopathological examination. Histopathological findings were noted and this was considered as gold standard for defining the outcome.

RMI was correlated with final histopathological report. The data was entered in an excel sheet. The $\mathrm{T}$ test for mean and the chi-Square test was used to compare the demographic, biochemical and USG data of subjects with benign and malignant adnexal masses.

The sensitivity, specificity and positive predictive value and negative predictive value of all the four RMI were calculated and data analyzed.

\section{RESULTS}

A total of 65 patients were included in the study. On the basis of histopathological examination, 40 subjects were 
diagnosed with benign condition, 4 had borderline and 21 had malignant tumour as can be seen in Table -2 . Malignant tumor included 8 cases of mucinous cystadenocarcinoma (12.3\%), 7 of serous cystadenocarcinoma (10.8\%), 3 of granulosa cell tumour (4.6), 2 of krukenberg's tumour (3.07\%) and 2 of malignant round cell tumours $(3.07 \%)$. Borderline malignancy included 3 cases of mucinous cystadenocarcinoma $(4.6 \%)$ and 1 case of serous cystadenocarcinoma (1.5\%). Benign Gynaecological conditions included 10 cases of mature cystic teratoma (15.38\%), 10 of serous cystadenoma (15.3\%), 3 of mucinous cystadenoma $(4.6 \%), 3$ of Endometriosis (4.6\%), 2 of fibroma (3.07\%), 1of fibrothecoma (1.5\%) 2 of tuboovarian abscess $(3.07 \%), 3$ of functional ovarian cyst $(4.6 \%), 1$ of struma ovari $(1.5 \%)$ and 1 of pseudomyxomaperitonei $(1.5 \%)$.

\section{Table 1: Scoring system based on USG findings.}

\begin{tabular}{|ll|}
\hline $\begin{array}{l}\text { USG findings } \\
\text { Unilocular simple cysts with regular fine wall or } \\
\text { lesions suggesting dermoid cyst }\end{array}$ & 0 \\
\hline $\begin{array}{l}\text { Multilocular cyst with regular and smooth wall } \\
\text { <3mm or thick 3mm or solid homogenous tumour } \\
\text { with hyperecogenic and well defined wall }\end{array}$ & 01 \\
\hline $\begin{array}{l}\text { Unilocular cyst or multilocular cyst with fine } \\
\text { wall with irregularity in the wall or septa >3 mm }\end{array}$ & 02 \\
\hline $\begin{array}{l}\text { Multilocular cyst with thick and irregular wall } \\
\text { (irregularily } 3 \mathrm{~m} \text { ) }\end{array}$ & 04 \\
\hline $\begin{array}{l}\text { Complex lesion, with predominancy of cystic or } \\
\text { solid area without irregularity in surface }\end{array}$ & 05 \\
\hline $\begin{array}{l}\text { Complex lesion with irregularity in surface } \leq \\
\text { 3mm or badly defined and irregular wall or solid } \\
\text { heterogeneous lesion. }\end{array}$ & 10 \\
\hline Multiplicity-unilateral lesions or bilateral lesion & 01 \\
\hline $\begin{array}{l}\text { Associated lesions ascites, wall expansive } \\
\text { involvement greater than 3mm }\end{array}$ & 02 \\
\hline
\end{tabular}

The distribution of benign and malignant cases according to age menopausal status, USG score, serum CA-125 and various RMI score is shown in Table -3 . The age of patients ranged from 19 to 70 years with mean (SD) being 52 years. Out of these, $72 \%$ of malignant cases were in age group 41 to 70 years of age. It was observed that $64 \%$ of malignant cases belonged to postmenopausal stage. The association between USG score and disease status was not statistically significant $(\mathrm{P}$ value $>0.05$ ). The association between tumour size and disease status was found to be statistically significant ( $\mathrm{P}$ value $<0.05$ ). Increasing CA-125 value in the subject with malignant disease showed statistically significant $P$ value of <0.0037. Results of univariate analysis showed a significant linear trend for malignancy according to postmenopausal status, large tumour size and increasing RMI value. The association between age, USG score and diseases status was not significant.

RMI 1,2,3,4 was calculated according to their formula. Data for sensitivity, specificity, positive and negative predictive value of different RMI is shown in Fig-1. Sensitivity of RMI- $1,2,3$ and 4 was calculated to be $63.63 \%, 77.27 \%, 63.63 \%$ and $77.27 \%$ respectively. Specificity of RMI- $1,2,3$ and 4 was calculated to be $69.04 \%, 64.28 \%, 64.28 \%$ and $62.79 \%$ respectively. It was observed that RMI-2 and RMI-4 had maximum sensitivity while RMI-1 had maximum specificity.

Table 2: Distribution of patients on basis of histopathological diagnosis.

\begin{tabular}{|lll|}
\hline Histopathological diagnosis & Number & Percentage \\
\hline Malignant & 21 & $32.3 \%$ \\
\hline Serous cystadenocarcinoma & 7 & 10.8 \\
Mucinous cystadenocarcinoma & 8 & 12.3 \\
Granulosa cell tumour & 3 & 4.6 \\
Krukenberg's tumour & 2 & 3.07 \\
Malignant round cell tumour & 2 & 3.07 \\
\hline Borderline malignancy & 4 & $6.2 \%$ \\
\hline Mucinous cystadenocarcinoma & 3 & 4.6 \\
\hline Serous cystadenocarcinoma & 1 & 1.5 \\
\hline Benign & 40 & $61.5 \%$ \\
\hline Mature cystic teratoma & 10 & 15.4 \\
Serous cystadenoma & 10 & 15.4 \\
Mucinous cystadenoma & 3 & 4.6 \\
Endometriosis & 3 & 4.6 \\
Fibroma & 2 & 3.07 \\
Fibrothecoma & 1 & 0.5 \\
Tuboovarian abscess & 2 & 3.07 \\
Functional cyst & 3 & 4.6 \\
Struma ovary & 1 & 1.5 \\
Pseudomyxomaperitonei & 1 & 1.5 \\
\hline
\end{tabular}

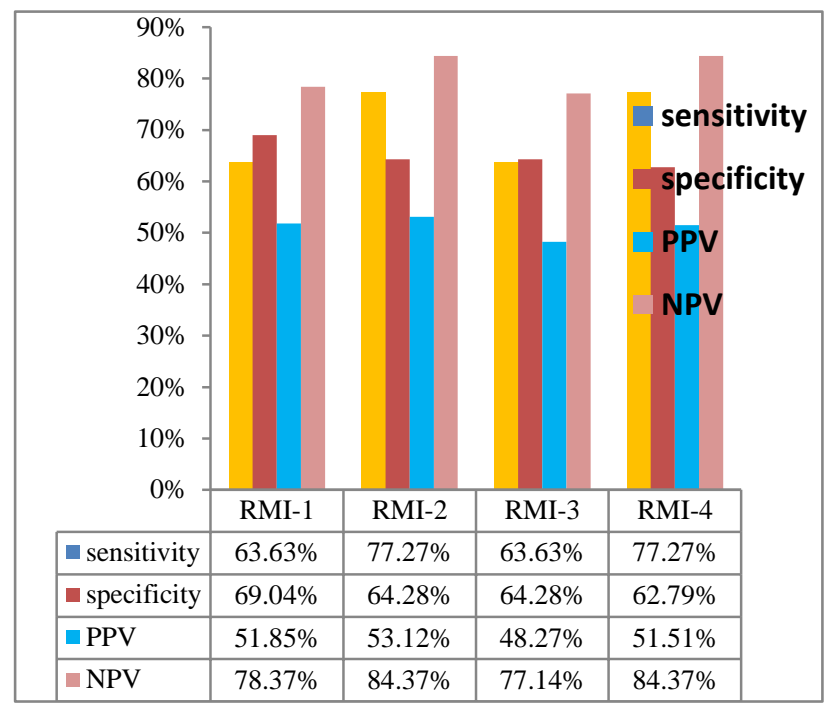

Figure 1: Statistical values of RMI 1, 2, 3 and 4.

\section{DISCUSSION}

Ovarian cancer has emerged as one of the most common gynaecological malignancies affecting women. Symptoms of the ovarian cancer are very vague that is why it is also known as 'silent killer'. Mortality rate due to ovarian cancer was highest among gynaecological 
malignancies. ${ }^{5}$ It is due to silent occurrence, slow progression and no effective methods for early diagnosis. Malignant tumours should be referred to specialized centres for gynaecological oncologic surgeries. Therefore differential diagnosis as benign and malignant tumours is essential in order to decide on the optimal approach in each case. To reduce the diagnostic dilemma between benign and malignant ovarian masses, a formula based scoring system known as risk of malignancy index (RMI) was introduced. The probability of malignant pelvic masses is calculated by incorporating serum CA-125 level, USG morphology of the pelvic masses and menopausal status.

Four RMI have been devised each with some different characteristics. RMI-1 was the first version developed by Jacob et al. ${ }^{2}$ When the ultrasound score was 0 , in RMI -1 $\mathrm{U}$ was made 0 resulting in RMI- 1 of 0 regardless of the CA 125 level. RMI-2 was developed by Tingulstat et al. They modified it to form the RMI-3. ${ }^{3}$ RMI- 2, 3, 4 had USG score 1-4. RMI-4 was created by Yamamoto et al that included tumor size as an additional parameter. ${ }^{4}$

Table 3: Distribution of benign and malignant cases in relation to age, menopausal status, USG score, serum CA125 and RMI-1, 2, 3, 4.

\begin{tabular}{|c|c|c|c|c|}
\hline Age & Benign & Borderline and Malignant & Chi square test & P value \\
\hline$\leq 50$ & 25 & 13 & \multirow{2}{*}{$X^{2}=0.698$} & $P>0.05$ \\
\hline$\geq 50$ & 15 & 12 & & Non-significant \\
\hline \multicolumn{5}{|c|}{ Menopausal status } \\
\hline Pre & 28 & 9 & \multirow{2}{*}{$X^{2}=7.25$} & $\mathrm{P}<0.01$ \\
\hline Post & 12 & 16 & & Significant \\
\hline \multicolumn{5}{|c|}{ USG Score } \\
\hline$\leq 1$ & 10 & 4 & \multirow{2}{*}{$X^{2}=7.37$} & $\mathrm{P}<0.05$ \\
\hline$\geq 7$ & 30 & 21 & & Non-significant \\
\hline \multicolumn{5}{|c|}{ Tumour Size } \\
\hline$\leq 7$ & 15 & 10 & \multirow{2}{*}{$X^{2}=0.0406$} & $\mathrm{P}<0.05$ \\
\hline$\geq 7$ & 25 & 15 & & Significant \\
\hline \multicolumn{5}{|c|}{ RMI 1, 2, 3} \\
\hline$>200$ & 14 & 15 & \multirow{2}{*}{$X^{2}=3.891$} & $\mathrm{P}<0.05$ \\
\hline$<200$ & 26 & 10 & & Significant \\
\hline \multicolumn{5}{|c|}{ RMI 4} \\
\hline$>450$ & 25 & 15 & \multirow{2}{*}{$X^{2}=0.0406$} & $\mathrm{P}<0.05$ \\
\hline$<450$ & 15 & 10 & & Significant \\
\hline \multicolumn{5}{|c|}{ CA -125 } \\
\hline$>35$ & 11 & 16 & \multirow{2}{*}{$X^{2}=8.440$} & $\mathrm{P}<0.0037$ \\
\hline$<35$ & 29 & 9 & & Significant \\
\hline
\end{tabular}

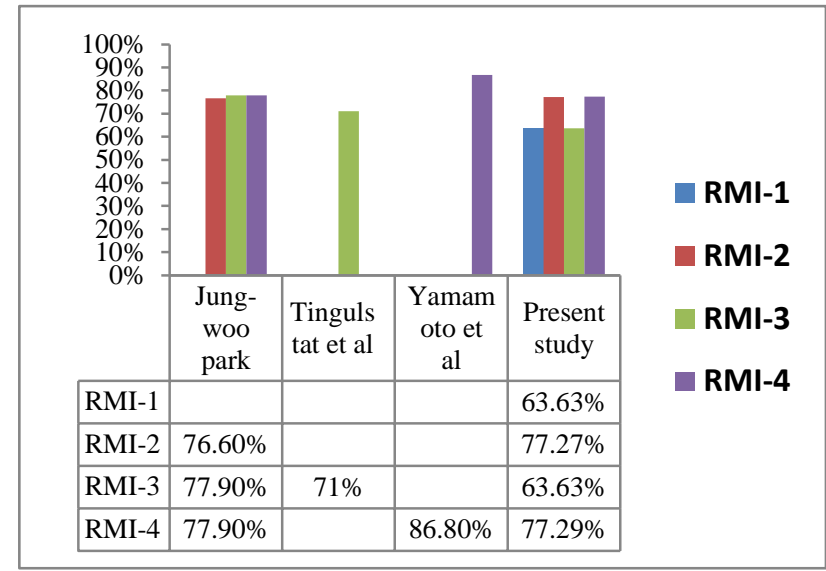

Figure 2: Comparison of sensitivity of RMI in different studies.



Figure 3: Comparison of specificity of RMI in different studies. 
The aim of this observational study was to evaluate the role of RMI- 1, 2, 3 and 4 in distinguishing benign from malignant adnexal masses. Sixty five cases were segregated over a period of 2 years. Of them $61.5 \%$ were benign, $32.3 \%$ were malignant and $6.2 \%$ where borderline on histopathological examination. In the study by Javdehal $\mathrm{R}$ et al $29.3 \%$ were malignant on histopathology including $3.4 \%$ borderline tumour. ${ }^{6}$ In our study, all the subjects with malignant disease underwent primary cytoreductive surgery and $60 \%$ of them received chemotherapy.

In the present study statistically significant relationship of malignant tumours was found with menopausal status, tumour size and CA-125. Age specific incidence rate found that ovarian cancer increases from 35 years and reaches a peak between 55 and 64 years. The factors associated with an increased risk include older age, race (white), nulliparity and family history of ovarian, endometrial or breast cancer.

In the present study, no significant association was found with age and USG score. However, Jung Woo Park found statistically significant association.

In our study univariate analysis showed a significant linear trend for malignancy according to post-menopausal status, large tumour size, RMI score $>200$ and increasing CA-125 level. Similar results were also found by Javdekar R et al in their study. ${ }^{6}$

In the present study, sensitivity, specificity, PPV and NPV of all four RMI was also calculated. It was observed that RMI-2 and RMI-4 had maximum sensitivity while RMI-1 had maximum specificity. Javdekar $\mathrm{R}$ et al showed RMI-2 to have maximum sensitivity. ${ }^{6}$

As seen in Figure 2 and 3, our study calculated sensitivity, specificity, PPV and NPV of all four RMI unlike other studies in which accuracy of one of the RMI was calculated. ${ }^{6,7}$ In the study of Jung Woo Park, statistical analysis for RMI 2, 3 and 4 were done but was not done for RMI- 1 . $^{7}$

In conclusion, we found that Risk of malignancy index is a good diagnostic tool to differentiate between benign and malignant pelvic masses. RMI- 2 and RMI-4 had maximum sensitivity while RMI-1 had maximum specificity. Overall RMI-2 appears to be the most accurate of all the four RMI.

\section{CONCLUSION}

There was a significant increase in CS rates over the last decade in Sikkim. Increased rates of primary CS have led to an increased proportion of the obstetric population with a history of previous caesarean deliveries. Another significant contributory factor in increasing proportion of CS were cesarean sections on maternal request without any co-existing medical or obstetric indication which appeared as second most common indication for elective cesarean section in recent years.

Funding: No funding sources Conflict of interest: None declared

Ethical approval: The study was approved by the Institutional Ethics Committee

\section{REFERENCES}

1. Nandagudi S, Murthy SS, Suman G. Changing Trends in Incidence of Ovarian Cancer - the Indian Scenario. Asian Pacific Journal of Cancer Prevention. 2009:10:1025-30.

2. Jacobs I, Oram D, Fairbanks J, Turnes J, Frost C, Grudzinskas JG. A risk of malignancy index incorporating CA125, Ultrasound and menopausal status for the accurate preoperative diagnosis of ovarian cancer. Br J obstet Gynaecol. 1990:97:922-9.

3. Tingulstad S, Hagen B, Shjerdestad FE, Onsrud M, Kiserud T, Halorsen T, et al . Evaluation of a risk of Malignancy index based on serum CA 125, USG findings and menopausal status in the preoperative diagnosis of pelvic masses. Br J obstet Gynaecol. 1996:103:826-31

4. Yamamoto, Yamada R, Oguri H, Maeda N, Fukaya T. Comparison of four malignancy risk indices in the preoperative evaluation of patients with pelvic masses. Em J Obstet Gynecol Reprod Biol. 2009;144:162-7.

5. Rossing MA, Wicklund KG, Cushing-Haugen KL. Predictive value of symptoms for early detection of ovarian cancer. J Natl CancerInst. 2010;102(4):2229.

6. Javdekar R, Maitra N. Risk of Malignancy Index (RMI) in Evaluation of Adnexal mass. The Journal Obstetrics and Gynaecology of India. 2015;65(2):117-21.

7. Jung-woo Park MD, Jee-Hyun Park ESS. Four risk of malignancy indices in evaluation of pelvic masses. Korean J Obstet Gynacol. 2012:55(9):636-43.

Cite this article as: Kumari N, Gupta V, Kumari R, Makhija A. Evaluation of risk of malignancy index as a diagnostic tool in cases with adnexal mass Int $\mathbf{J}$ Reprod Contracept Obstet Gynecol 2016;5:1857-61. 